\title{
Challenges of Urban Sensing
}

\author{
Henry Tirri \\ Nokia Research Center \\ Henry.Tirri@nokia.com
}

\begin{abstract}
Wireless sensor networks are emerging as a critical information technology, and they are continuing the trend originating in mainframe computing currently at the stage of mobile computing. This trend shows several aspects consistent in the evolution of computing including the increasing hardware miniaturization of the computing units and an increasing emphasis of the role of communication between the computing units - "networking". In addition from the software side there is an increasing need to software solutions that are robust, exhibit distributed control, collaborative interfaces resulting in adaptive capabilities also at the system level. Like the present Internet, wireless sensor networks are large-scale distributed systems, but composed of smart sensors and actuators. They will eventually infuse the physical world and provide "grounding" for the Internet thus creating the Internet of Things. Research on wireless sensor networks has been taking place at several levels, from the lowest physical level to the highest information level - the latter is much less developed than the research at the physical levels. In addition, much of the research in wireless sensor networks has been focusing on military or science applications. However, wireless sensor networks can also play an important role in the realization of ubiquitous computing for everyday life - creating what we call "Urban sensing environment". In urban sensing many natural gateways exist to collect and process the sensor information - static ones such as media devices, or mobile devices such as smart phones that can collect sensor information when entering the communication range of an active sensor. Some of the applications of wireless sensor network technology at home include, in addition to the surveillance functions, adding "intelligence" to utility consumption, electronic tagging, contamination control and disaster monitoring. Similarly at the community level "traffic monitoring" including people allows a development of totally unseen services from micro weather forecasts to new ways for "sensing the environment" for entertainment. In this talk we will outline some of the research challenges for urban sensing, and the role of learning and data analysis techniques for solving those challenges.
\end{abstract}

